\title{
Validación transcultural del HypoA-Q para medir conciencia de hipoglucemia en pacientes diabéticos
}

\section{Cross-cultural validation of the HypoA-Q to measure hypoglycemic awareness in diabetic patients \\ Validação transcultural do HypoA-Q para mensurar consciência de hipoglicemia em pacientes diabéticos}

\author{
Ana Julia Carrillo Algarra ${ }^{1}$ \\ Sandra Milena Hernández-Zambrano ${ }^{2}$ \\ Fabio Alexander Sierra-Matamoros ${ }^{3}$ \\ Diana Cristina Henao-Carrillo ${ }^{4}$ \\ Ana María Gómez-Medina ${ }^{5}$ \\ Daniel Esteban Hurtado-Barrera ${ }^{6}$
}

\section{Resumen}

Introducción: La hipoglucemia inadvertida incrementa el riesgo de la hipoglucemia severa en pacientes con diabetes. Una medición objetiva de la misma requiere instrumentos como el HypoA-Q, que, a diferencia de otros, mide conciencia de episodios nocturnos; sin embargo, no se dispone de una versión en español. Objetivo: Traducir y adaptar culturalmente la escala HypoA-Q para la medición de conciencia de la hipoglucemia en pacientes colombianos con Diabetes Mellitus. Metodología: Estudio de Adaptación transcultural del instrumento HypoA-Q. Se obtuvo la autorización de la autora para su traducción y adaptación a la población colombiana. Se siguió la metodología de la European Organisation for Research and Treatment for Cancer (EORTC). Al respecto, se realizaron dos traducciones directas por hablantes nativos del español, una versión reconciliada, dos traducciones inversas por dos hablantes nativos del inglés y una prueba piloto en 15 pacientes con diagnóstico de diabetes. Resultados: Aunque las traducciones directas al español fueron similares, se hicieron ajustes para obtener una versión reconciliada con palabras más fáciles de comprender cambiando las unidades de $\mathrm{mmol} / \mathrm{L}$ a $\mathrm{mg} / \mathrm{dl}$. Las traducciones inversas fueron similares al original en inglés. La prueba piloto, realizada en 15 pacientes, no requirió ajustar ningún ítem, pues la frecuencia de ítems con dificultades de comprensión, confusión o palabras ofensivas fue menor al 15\%. Conclusiones: Se dispone de la versión en español para Colombia de la escala HypoA-Q. Se aconseja realizar un estudio de evaluación de sus propiedades psicométricas antes de ser usada en investigaciones o en la práctica clínica.

Palabras Clave: Hipoglucemia; Diabetes mellitus; Conciencia; Encuestas y cuestionarios; Estudios de validación.
Autor de correspondencia*

1* Enfermera, Mg. en Administración en Salud. Profesora Titular, Facultad de Enfermería. Grupo Perspectivas del Cuidado. Fundación Universitaria de Ciencias de la Salud. Bogotá, Colombia. Correo: ajcarrillo@fucsalud.edu. co. (D) 0000-0002-3765-7474

${ }^{2}$ Enfermera, PhD en Ciencias de la Salud, Mg. en cuidados de salud para la promoción de la autonomía de las personas y la atención a los procesos de fin de vida. Docente Investigadora, Facultad de Enfermería. Grupo Perspectivas del Cuidado. Fundación Universitaria de Ciencias de la Salud. Bogotá, Colombia. Correo: smhernandez3@fucsalud. edu.co. (i) 0000-0001-5504-3065

Recibido: 11 junio 2021

Aprobado: 12 noviembre 2021

Para citar este artículo

Carrillo-Algarra AJ, Hernázdez-Zambrano SM, Sierra-Matamoros FA, Henao-Carrillo DC, Gómez-Medina AM, Hurtado-Barrera DE. Validación transcultural del HypoA-Q para medir conciencia de hipoglucemia en pacientes diabéticos. Rev. cienc. cuidad. 2022; 19(1):42-56. https://doi. org/10.22463/17949831.3056

(C) Universidad Francisco de Paula Santander. Este es un artículo bajo la licencia CC-BY-NC-ND is to translate and culturally adapt the HypoA-Q in order to be able to measure hypoglycemia awareness in Colombian patients with diabetes. Methodology: It is a cross-cultural adaptation study of the HypoA-Q instrument. Authorization was obtained from the author for its trans-

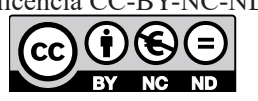




\section{Cienciäs Cuidado}

Scientific Journal of Nursing lation and adaptation to the Colombian population. The European Organization for Research and Treatment of Cancer (EORTC) methodology was followed. The researchers obtained a reconciled Spanish version with simple vocabulary. Two direct translations were performed by native Spanish speakers, one reconciled version, two reverse translations by two native English speakers, and a pilot test in 15 patients diagnosed with diabetes. Results: The direct translations into Spanish were similar. Adjustments were made to obtain a reconciled version with simple words. Also, glucose units were changed from $\mathrm{mmol} / \mathrm{L}$ to $\mathrm{mg} / \mathrm{dl}$. The reverse translations were similar to the original in English. 15 patients older than 18 years read the Spanish version of HypoA-Q and answered a questionnaire to verify if each of the items were understandable, confusing, annoying or if it had difficult words. It did not require adjusting any item, since the frequency of items with comprehension difficulties, confusion or offensive words was less than $15 \%$. Conclusions: The Spanish version of the HypoA-Q is available. An evaluation study of its psychometric properties must be carried out before being used in research studies or in clinical practice.

Key words: Hypoglycemia; Diabetes mellitus; Awareness; Surveys and questionnaires; Validation study

\section{Resumo}

Introdução: A hipoglicemia inadvertida incrementa o risco de hipoglicemia severa em pacientes com diabetes. Uma medição da mesma requer instrumentos como o HypoA-Q, que a diferença de outros, mede consciência de episódios noturnos; entretanto, não se dispõe de uma versão em espanhol. Objetivo: Traduzir e adaptar culturalmente a escala HypoA-Q para mensurar a consciência de hipoglicemia em pacientes colombianos com Diabetes Mellitus. Materiais e métodos: estudo de adaptação transcultural do instrumento HypoA-Q. Obteve-se a autorização da autora para a sua tradução e adaptação à população colombiana. Seguiu-se a metodologia da European Organization for Research Treatment for Cancer (EORTC). Realizaram-se duas traduções diretas por falantes nativos de língua espanhola e uma versão conciliada, duas traduções inversas por falantes nativos da língua inglesa e uma prova piloto em 15 pacientes com diagnostico de diabetes. Resultados: Embora as traduções diretas ao espanhol foram semelhantes, foram feitos ajustes para obter uma versão conciliada com palavras mais simples de compreender substituindo as unidades de $\mathrm{mmol} / \mathrm{L}$ para $\mathrm{mg} / \mathrm{dl}$. As traduções inversas foram semelhantes ao instrumento original em inglês. A prova piloto realizada não precisou ajustar nenhum item, devido a que os itens com dificuldade de compreensão, confusão ou palavras ofensivas foi inferior ao $15 \%$. Conclusão: Dispõe-se da versão em espanhol para Colômbia da escala HypoA-Q. Aconselha-se realizar um estudo de avaliação das propriedades psicométricas antes de ser usada em pesquisa ou na prática clinica.

Palavras-chave: Hipoglicemia; Diabetes Mellitus; Consciência; Inquéritos e questionários; Estudo de validação.
${ }^{3}$ Psicólogo, Mg. en Epidemiología Clínica. Instructor asistente, Facultad de Medicina, Fundación Universitaria de Ciencias de la Salud - Hospital de San José. Bogotá, Colombia. Correo: fasierra@ fucsalud.edu.co (iD) 0000-00029996-2431.

4 Médica especialista en Endocrinología. Facultad de medicina Pontificia Universidad Javeriana Hospital de San Ignacio. Bogotá Colombia. Correo: fucshenao@ hotmail.com. (1) 0000-0002-1353148X.

5 Médica especialista en Endocrinología. Facultad de medicina Pontificia Universidad Javeriana Hospital de San Ignacio. Bogotá, Colombia. Correo: amgomezm5@ gmail.com. (D) 0000-0002-89073470 .

${ }^{6}$ Enfermero de la Facultad de Enfermería. Fundación Universitaria de Ciencias de la Salud. Bogotá, Colombia. Correo: dehurtado@ fucsalud.edu.co. (D) 0000-00027938-6510.

\section{Introducción}

Actualmente, la diabetes mellitus (DM) es considerada una de las enfermedades crónicas no transmisibles de mayor prevalencia en el mundo. Colombia, según la
Cuenta de Alto Costo en el 2018 reportó 1.294.940 personas con diagnóstico de diabetes mellitus, con mayor prevalencia en el sexo femenino con 2,58 casos nuevos por cada 100 habitantes (1).

El cuidado de la salud de pacientes diabéticos tratados 


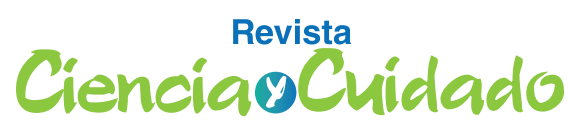

Scientific Journal of Nursing

con insulina requiere prevenir la hipoglucemia, ya que es la complicación más frecuente (2-6) y constituye el principal obstáculo para lograr metas de control glucémico. La hipoglucemia se clasifica según las consecuencias clínicas en: severa, cuando hay pérdida del estado de conciencia o requiere ayuda de otra persona para controlarla; clínicamente significativa, cuando la glucosa capilar es menor de $54 \mathrm{mg} / \mathrm{dl}$ medida por glucometría capilar o el mismo valor durante 20 minutos medido por Monitoreo Continuo de Glucosa; alerta de hipoglucemia, es el caso en que los síntomas se acompañan de medición de glucosa inferior a $70 \mathrm{mg} / \mathrm{dl}$; asintomática si no hay síntomas clínicos pero la medición de glucosa es inferior a $70 \mathrm{mg} / \mathrm{dl}$; relativa, cuando el paciente presenta síntomas, se los interpreta como tal pero la glucosa es mayor de $70 \mathrm{mg} / \mathrm{dl}(7,8)$.

Por otra parte, estudios en la vida real han documentado que la hipoglucemia severa es frecuente en pacientes con diagnóstico de diabetes mellitus tipo 2; en el caso del subgrupo de Colombia se presentó en el 65,7\% de los pacientes reclutados (9). Estudios recientes asocian la incidencia de la hipoglucemia severa con el incremento de mortalidad global y cardiovascular (10-16), razón por la cual, se deben identificar factores de riesgo, como edad avanzada, insuficiencia renal, alta variabilidad glucémica y percepción anormal de síntomas de hipoglucemia o hipoglucemia asintomática. Algunos pacientes, dada la recurrencia de episodios de hipoglucemia no grave, pierden la capacidad de identificar sus síntomas, generando hipoglucemias inadvertidas que multiplican el riesgo de la hipoglucemia grave (17-19). Se puede señalar que existen diferentes instrumentos para medir la conciencia de hipoglucemia (17) como el puntaje de Gold score, que consiste en un autoinforme breve que contiene una pregunta única, reporta buena validez de apariencia pero dificulta la caracterización de la hipoglucemia inadvertida (20); también, están el Cuestionario de Clarke, validado en español, mide el umbral glucémico y la respuesta sintomática a la Hipoglucemia $(21,22)$ y el método de Pedersen-Bjergaard (23) que sobrestima la prevalencia de hipoglucemias indetectables y no debe ser utilizado en el contexto clínico. Pocos han sido validados en pacientes con diabetes mellitus tipo 2 .

De acuerdo con lo planteado anteriormente es necesario utilizar un instrumento que mida la conciencia de la hipoglucemia en pacientes diabéticos, por tanto el objeto del presente estudio es la validación transcultural del
Validación transcultural del HypoA-Q para medir conciencia de hipoglucemia en pacientes diabéticos

Instrumento HypoA-Q, que fue seleccionado en virtud de que mide la conciencia de la hipoglucemia cuando se está despierto y dormido, y evalúa tres subescalas que reflejan la conciencia alterada, el nivel de síntomas y su frecuencia. Además, el análisis psicométrico reporta validez de apariencia, validez de contenido, consistencia interna, y una estructura satisfactoria para valorar la conciencia de la hipoglucemia, constituyéndose en un instrumento de relevancia clínica, al caracterizar los episodios nocturnos de hipoglucemia, determinando su gravedad, frecuencia y utilización de servicios de salud para su tratamiento (24). Al respecto, se realizó un estudio de adaptación transcultural de la escala Hypo-AQ para su aplicación y uso en la población colombiana. Para garantizar la equivalencia entre la versión en inglés y español se efectuó un proceso de traducciones directas e inversas y una prueba piloto con el propósito de lograr la adaptación transcultural, siguiendo la metodología propuesta por el grupo de calidad de vida de la European Organisation for Research and Treatment of Cancer EORTC (25).

\section{Objetivos}

\section{Objetivo general}

Traducir y adaptar culturalmente la escala HypoA-Q para la medición de conciencia de hipoglucemia en pacientes colombianos con Diabetes Mellitus.

\section{Objetivos específicos}

- Obtener una versión en español de la escala HypoA-Q.

- Identificar problemas de comprensión, confusión o palabras ofensivas en la traducción.

- Evaluar y ajustar el parafraseo de la traducción.

\section{Métodos}

\section{Tipo de estudio}

Estudio de adaptación transcultural de un instrumento de medición. Se llevaron a cabo seis pasos:

1. Solicitud de autorización para la traducción y uso de la escala.

2. Realización de traducciones directas.

3. Elaboración de versión preliminar. 


\section{CiencianoCuidado}

Scientific Journal of Nursing

4. Elaboración de traducciones inversas.

5. Revisión del proceso y comparación con escala original.

6. Realización de prueba piloto.

\section{Instrumento}

El cuestionario de conciencia de la hipoglucemia (HypoA-Q) es una escala que consta de 20 ítems (33 si se consideran de manera independiente las opciones de respuesta para algunos ítems), la mayoría en escala tipo Likert que evalúan cómo el paciente recuerda: los episodios de hipoglucemia (frecuencia, severidad), la utilización de medidas para cuidar su propia salud, umbrales de glucosa en sangre cuando se presentaron los síntomas, la conciencia de los síntomas, la conciencia alterada y la frecuencia de monitorización de glucosa en sangre al momento de "sentirse mal". Igualmente, identifica síntomas de hipoglucemia experimentados mientras los pacientes están despiertos o dormidos. La HypoA-Q es una medición de perfil, por ello da como resultado varios puntajes de ítems y sub-escalas. Se debe precisar que los usuarios pueden seleccionar los ítems o sets de ítems según sus propósitos. Dieciséis ítems se

relacionan con el reporte de eventos hipoglicémicos y la utilización de medidas para cuidar su propia salud. La distribución de los ítems es la siguiente:

- Conciencia de hipoglucemia en general (ítems $1,2 \mathrm{a}-2 \mathrm{~d})$

- Conciencia de hipoglucemia mientras está despierto (ítems 3, 4a-4d)

- Conciencia de hipoglucemia mientras está durmiendo (ítems 15, 16a-16e) (24).

\section{Procedimiento}

Se realizó un estudio de traducción y adaptación transcultural de la versión original en inglés del instrumento HypoA-Q (24), siguiendo la metodología propuesta por la EORTC (25) a fin de obtener una versión en español a ser utilizada en Colombia. Para esto se conformó un grupo de trabajo (equipo investigador) en el que participaron: dos médicas endocrinólogas, una enfermera doctora en ciencias de la salud, una enfermera magíster en administración de salud, un psicólogo magíster en epidemiología clínica y un estudiante de enfermería. El proceso incluyó los pasos ilustrados en la figura 1.

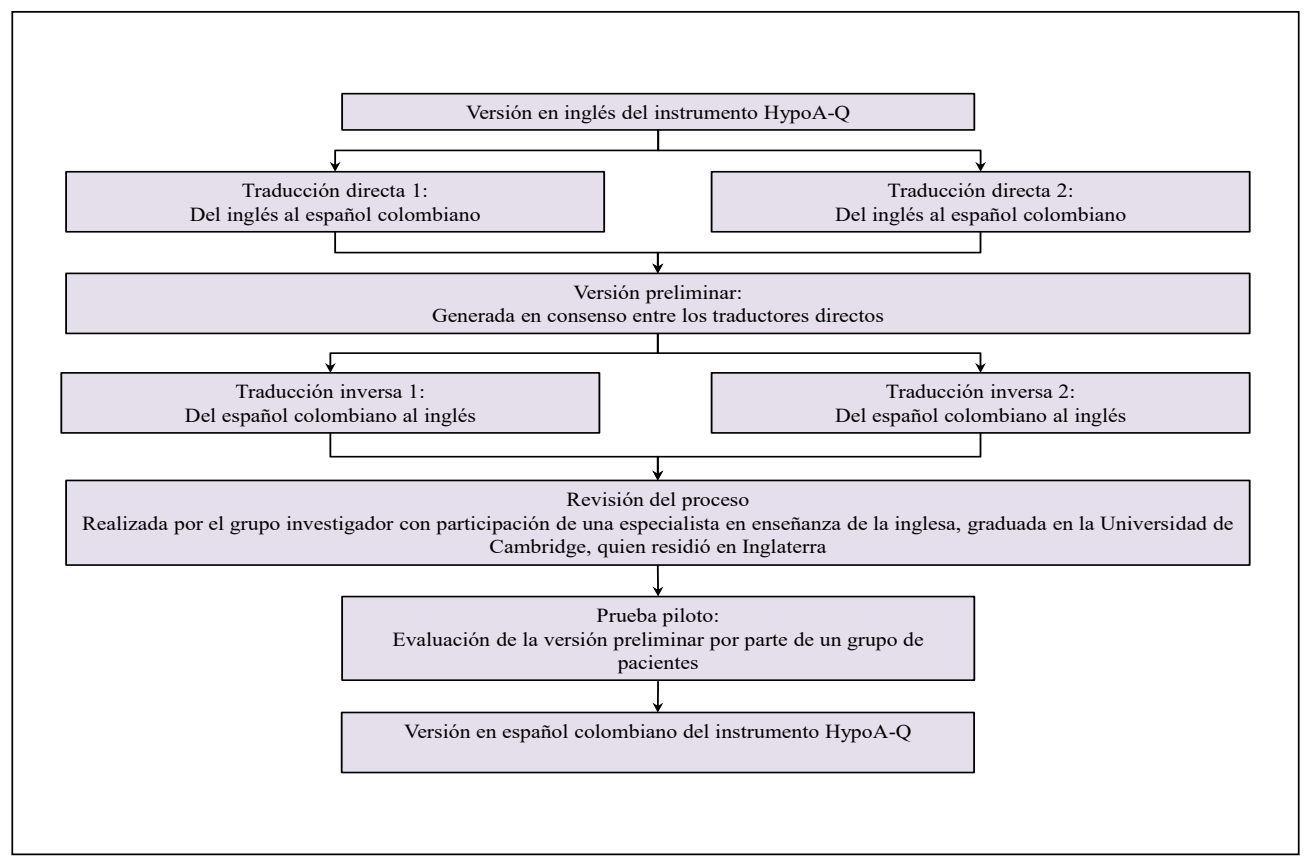

Figura 1. Proceso de traducción y adaptación cultural de la escala HypoA-Q.

Fuente: Elaborado por los investigadores. 


\section{CiencianoCuidado}

Scientific Journal of Nursing

Paso 1. Solicitud de autorización para la traducción y uso de la escala

La escala y el permiso para su traducción y validación fueron solicitados a su autora la doctora Jane Speight, quien autorizó por escrito al grupo investigador.

\section{Paso 2. Realización de traducciones directas}

Se realizaron dos traducciones de la escala del inglés al español colombiano, de manera independiente, por dos médicos especializados en medicina interna, hablantes nativos del español con buena fluencia del inglés y experiencia en el tratamiento de pacientes con diabetes.

\section{Paso 3. Elaboración de versión preliminar}

A partir de las dos traducciones directas el equipo investigador obtuvo una versión preliminar, para ello escogió la mejor traducción y elaboró una a partir de las anteriores de acuerdo con los siguientes criterios:

- Comprensión. Que refleja definiciones conceptuales del texto original, comprensibles para personas sin conocimiento médico y diferentes niveles educativos, no requiriese información adicional y se leyera naturalmente en español.

- Cultura. La más apropiada en temas sensibles y que utilizara términos acordes con la cultura colombiana.

- Gramática. Con sintaxis correcta en formas y tiempos verbales, género, número uso de artículos y preposiciones.

- Terminología. Que incluyera todas las palabras clave y emplea vocabulario consistente a través de todo el texto (25).

\section{Paso 4. Elaboración de traducciones inversas}

La versión preliminar se envió a dos expertos nativos de lengua inglesa, con buena fluencia del español, certificados por empresas dedicadas a traducciones, quienes, de manera independiente sin conocer la escala original, realizaron la traducción inversa al inglés.

\section{Paso 5. Revisión del proceso y comparación con escala original}

El equipo investigador con la asesoría de una especialista en enseñanza de la lengua inglesa de la Universidad
Validación transcultural del HypoA-Q para medir conciencia de hipoglucemia en pacientes diabéticos de Cambridge, comparó las dos traducciones inversas con la escala original para determinar si existía similitud de gramática y significado. Cuando se observó alguna discrepancia entre las versiones inversas y la original, se revisó y modificó la traducción preliminar en español.

\section{Paso 6. Realización de prueba piloto}

La escala con los ajustes propuestos en el paso anterior fue presentada a un grupo de 15 pacientes colombianos, mayores de 18 años, diagnosticados con Diabetes Mellitus tipo 1 y 2, quienes se encontraban recibiendo tratamiento o asistían a consulta de control en el Hospital San José. Los pacientes leyeron la escala $\mathrm{y}$ respondieron un cuestionario para verificar si cada uno de los ítems junto con las instrucciones de la escala eran comprensibles, confusos, molestos o tenían palabras difíciles, lo que implicaba su redacción con un mejor parafraseo. Si alguna pregunta no cumplía estos criterios en un porcentaje mayor al $15 \%$, se la hizo un ajuste a la versión en español.

Consideraciones éticas: Este estudio tiene aprobación del comité de ética del Hospital San José con fecha de 4 de octubre del 2018. Se garantizó el anonimato y confidencialidad de los datos.

\section{Resultados}

\section{Resultados del proceso de traducción}

Las traducciones directas fueron similares en terminología y uso de tiempos verbales, número, artículos y preposiciones. Sin embargo, se presentaron las siguientes discrepancias:

- Una traducción usó el término hipoglucemia, la otra el término hipoglicemia. Se escogió hipoglucemia porque hipoglicemia no aparece en el diccionario de la lengua española (26).

- Una traducción usó el término glucemia baja, la otra, glucosa baja en sangre ("low blood glucose" en el apartado instrucciones). Se escogió la última opción por ser más fácil de entender para pacientes con distintas escolaridades y más fiel al original en inglés.

- Una traducción usó el término urgencias, la otra el término emergencias (el original en inglés es 
"hospital (A\&E)", en el ítem 2). Se escogió la segunda opción por ser más fiel al término original en inglés y más comprensible para los pacientes.

- La expresión "how often have you had any hypo" fue traducida como "con qué frecuencia presentó usted alguna hipoglicemia" y "con qué frecuencia presentó episodios de hipoglicemia"; sin embargo, se decidió usar una expresión que utilizara términos más fieles al original y fuese más comprensible para pacientes con distintas escolaridades, se tradujo como "qué tan a menudo ha sufrido hipoglucemia en la que:

- La expresión “...you needed help / were unable" (ítem 2) fue traducida como "...usted haya necesitado ayuda" y “...usted necesitó ayuda"; se escogió la última opción por emplear el mismo tiempo verbal de la escala original en inglés. Teniendo en cuenta que varios ítems fueron redactados en tiempo pasado, se prefirió la traducción con redacción en pretérito perfecto simple, antes que la expresión en pretérito perfecto compuesto.

- En los ítems 4 y 16 la expresión "to give you sugar by mouth" fue traducida como "darle azúcar por la boca" y "administrarle carga oral de azúcar". Dadas las diferencias entre las dos, se decidió utilizar una expresión de uso común en la práctica clínica y para los pacientes: "haya necesitado que alguien más le diera glucosa por vía oral" la cual es fácil de entender e incluye el concepto original de la escala.

- El término "pounding heart", se tradujo como "latidos cardíacos fuertes (palpitaciones)" y como "corazón acelerado". Se escogió la segunda opción por ser más comprensible para la población colombiana.

- El término "difficulty speaking" se tradujo como "alteración del habla", y como "dificultad para hablar"; se prefirió la última opción por ser más fácil de comprender para pacientes con distintos niveles de escolaridad y ser una expresión empleada con más frecuencia por ellos.

- El término "tingling", se tradujo como estremecimiento y como hormigueo. Se dejó la palabra cosquilleo en la versión preliminar por ser más usada por los pacientes.

- El ítem 8 ('I 'just know' when I am going hypo by the way that I feel") las traducciones fueron "Yo 'simplemente sé' que estoy entrando en hipoglicemia por la manera como me siento", y
"Yo 'simplemente sé' cuando voy en hipoglucemia por la manera en que me siento"; se utilizó a partir de las anteriores, una expresión de más fácil comprensión para los pacientes: "Yo simplemente sé" cuando estoy teniendo hipoglucemia por cómo me siento.

- El ítem 11 "I am less aware of my hypos coming on than I used to be", se tradujo como "Percibo menos que estoy entrando en hipoglucemia de lo que solía hacerlo" y como "Percibo menos las hipoglucemias que lo que solía percibirlas antes"; se modificaron estas traducciones y se redactó el ítem de la siguiente manera: "Ahora soy menos consciente de cuando empiezan mis hipoglucemias que antes".

- Los ítems. 17 y 19 utilizan el verbo "wake up" ("I have symptoms which wake me when my blood glucose is low"; "my insulin pump / monitoring device wakes me when my blood glucose is low". Una de las traducciones utilizó el verbo "levantarse", la otra el verbo "despertarse"; se escogió la opción despertarse por ser más fiel al concepto de la escala original.

- En el ítem 20 una traducción a la expresión "when you are asleep" fue "mientras se está dormido", la otra traducción fue "durante el sueño"; se escogió la primera por ser más comprensible en nuestro contexto.

Una vez resueltas las anteriores discrepancias, se ajustó el ítem 5 "In the past month, have you had blood glucose readings (in mmol/l) ..." cambiando la unidad de medición a $\mathrm{mg}(\mathrm{dL})$, registrando los valores de acuerdo con la equivalencia correspondiente, por ser la unidad de medida oficial para Colombia, así (27):

- $\quad 3.5$ a $3.9 \mathrm{mmol} / \mathrm{l}$ : entre 63 a $70 \mathrm{mg} / \mathrm{dl}$

- 3.0 a $3.4 \mathrm{mmol} / \mathrm{l}$ : entre 54 a $62 \mathrm{mg} / \mathrm{dl}$

- $\quad 2.5$ a $2.9 \mathrm{mmol} / \mathrm{l}$ : entre 45 a $53 \mathrm{mg} / \mathrm{dl}$

- menos de 2.5: menos de $45 \mathrm{mg} / \mathrm{dl}$

Con estos ajustes se construyó una versión preliminar que fue traducida al inglés por dos investigadores de manera independiente. Al comparar estas versiones con la original en inglés no se encontraron diferencias.

\section{Resultados de la prueba piloto}

Se incluyeron 15 pacientes, cuyas características se 
Scientific Journal of Nursing

presentan en la tabla 1. La edad promedio fue de 46 años (desviación estándar 16,7 años), la frecuencia de hombres y mujeres fue similar, la mayoría tenían peso normal o sobrepeso, escolaridad primaria y secundaria y manifestaron estar empleados. E1 53,3\% tenía diagnóstico de Diabetes Mellitus tipo 1 y el 46,7\% Diabetes mellitus tipo 2 ; el 46,7\% consumía tres o más medicamentos simultáneamente.

Tabla 1. Características demográficas y clínicas de los participantes en la prueba piloto

\begin{tabular}{|c|c|}
\hline Característica & $\mathbf{N}(\%)$ \\
\hline Edad - media (desviación estándar) & $46(16,7)$ \\
\hline Sexo femenino & $8(53,3)$ \\
\hline \multicolumn{2}{|l|}{ IMC } \\
\hline Normal & $7(46,7)$ \\
\hline Sobrepeso & $6(40,0)$ \\
\hline Obesidad & $2(13,3)$ \\
\hline \multicolumn{2}{|l|}{ Escolaridad } \\
\hline Primaria & $5(33,3)$ \\
\hline Secundaria & $6(40,3)$ \\
\hline Técnico & $1(6,7)$ \\
\hline Universitario & $3(20,0)$ \\
\hline \multicolumn{2}{|l|}{ Ocupación } \\
\hline Empleado & $7(46,7)$ \\
\hline Independiente & $3(20,0)$ \\
\hline Ama de casa & $3(20,0)$ \\
\hline Pensionado & $1(6,7)$ \\
\hline Estudiante & $1(6,7)$ \\
\hline Diabetes tipo I & $8(53,3)$ \\
\hline Diabetes tipo II & $7(46,7)$ \\
\hline \multicolumn{2}{|l|}{ Antecedentes } \\
\hline Ninguno & $9(60,0)$ \\
\hline Nefropatía & $4(26,6)$ \\
\hline Retinopatía & $3(19)$ \\
\hline Neuropatía & $1(7)$ \\
\hline \multicolumn{2}{|l|}{ Esquema de insulina } \\
\hline Basal & $10(66,7)$ \\
\hline Basal bolo & $5(33,3)$ \\
\hline \multicolumn{2}{|l|}{ Insulina de larga acción } \\
\hline Glargina & $10(76,9)$ \\
\hline Detemir & $1(7,7)$ \\
\hline Degludec & $1(7,7)$ \\
\hline Ninguna & $1(7,7)$ \\
\hline \multicolumn{2}{|l|}{ Insulina de corta acción } \\
\hline Aspart & $6(40,0)$ \\
\hline Glulisina & $3(20,0)$ \\
\hline Lispro & $3(20,0)$ \\
\hline Ninguna & $3(20,0)$ \\
\hline
\end{tabular}


CiencianoCuidado

Scientific Journal of Nursing
Polifarmacia

Medicamentos asociados a hipoglucemia

$\begin{array}{ll}\text { Aspirina } & 5(45,5) \\ \text { Beta-Bloqueadores (Labetalol, metoprolol, propanolol, carvedilol) } & 2(18,2) \\ \text { Levotiroxina } & 6(54,5) \\ \text { Diuréticos (Clorotiazida, hidroclorotiazida, furosemida) } & 3(27,3) \\ \text { Comorbilidades } & \\ \text { Endocrinológica } & 9(69,2) \\ \text { Cardiovascular } & 2(15,4) \\ \text { Gastrointestinal } & 2(15,4) \\ \text { Hematológica } & 1(7,7) \\ \text { Respiratoria } & 1(7,7) \\ \text { Ejercicio } & 5(33,3)\end{array}$

Fuente: Elaborado por los investigadores
$7(46,7)$

$5(45,5)$

$2(18,2)$

$(54,5)$
Las características de los participantes en la prueba piloto indican que la muestra es representativa de los pacientes con diabetes y fue heterogénea en cuanto a características sociodemográficas y clínicas, lo cual indica que la prueba de la versión preliminar se aplicó en personas con diferentes grados de escolaridad y distintos niveles de conciencia de la hipoglucemia, población objetivo de la escala.
La prueba piloto del instrumento HypoA-Q (Tabla 2) reportó que los ítems 2c, 2d, 3, 4a, 4b, 5, 8, 9, 10, 14, $1516 \mathrm{a}, 17,18$ y 20 no presentaron ninguna objeción o comentario; en los ítems 1, 2b, 4c, 6a, 6b, 6c, 7, 12, 13, $16 \mathrm{~b}, 16 \mathrm{~d}$ y $16 \mathrm{e}$ se encontró que menos del $15 \%$ de los pacientes tenía objeción o comentario como: "Definir Hipoglucemia", "Agruparía Preguntas", entre otros, que no requirieron modificación a la escala.

Tabla 2. Resultados de la prueba piloto: frecuencia de pacientes que manifestaron alguna dificultad con la versión preliminar.

\begin{tabular}{lllll}
\hline \multicolumn{1}{c}{ Ítems } & \multicolumn{1}{c}{$\begin{array}{c}\text { Comprensión } \\
\mathbf{n}(\mathbf{\%})\end{array}$} & \multicolumn{1}{c}{$\begin{array}{c}\text { Confusión } \\
\mathbf{n}(\%)\end{array}$} & $\begin{array}{c}\text { Uso de palabras } \\
\text { dificiles } \mathbf{n}(\%)\end{array}$ & $\begin{array}{c}\text { Uso de palabra } \\
\text { ofensivas n }(\%)\end{array}$ \\
\hline 1 & $2(13,3)$ & $1(6.7)$ & $1(6.7)$ & $0(0)$ \\
2 opción a & $0(0)$ & $1(6.7)$ & $0(0)$ & $0(0)$ \\
2 opción b & $1(6.7)$ & $0(0)$ & $0(0)$ & $0(0)$ \\
2 opción c & $0(0)$ & $0(0)$ & $0(0)$ & $0(0)$ \\
2 opción d & $0(0)$ & $0(0)$ & $0(0)$ & $0(0)$ \\
3 & $0(0)$ & $0(0)$ & $0(0)$ & $0(0)$ \\
4 opción a & $0(0)$ & $0(0)$ & $0(0)$ & $0(0)$ \\
4 opción b & $0(0)$ & $0(0)$ & $0(0)$ & $0(0)$ \\
4 opción c & $2(13,3)$ & $1(6.7)$ & $1(6.7)$ & $0(0)$ \\
4 opción d & $8(53,3)$ & $3(20,0)$ & $9(60,0)$ & $0(0)$ \\
5 opción a & $0(0)$ & $0(0)$ & $0(0)$ & $0(0)$ \\
5 opción b & $0(0)$ & $0(0)$ & $0(0)$ &
\end{tabular}




\section{CienciagoCuidado}

Scientific Journal of Nursing

\begin{tabular}{lllll}
5 opción c & $0(0)$ & $0(0)$ & $0(0)$ & $0(0)$ \\
5 opción d & $0(0)$ & $0(0)$ & $0(0)$ & $0(0)$ \\
6 opción a & $1(6.7)$ & $0(0)$ & $0(0)$ & $0(0)$ \\
6 opción b & $1(6.7)$ & $0(0)$ & $0(0)$ & $0(0)$ \\
6 opción c & $1(6.7)$ & $1(6.7)$ & $0(0)$ & $0(0)$ \\
7 & $1(6.7)$ & $0(0)$ & $0(0)$ & $0(0)$ \\
8 & $0(0)$ & $0(0)$ & $0(0)$ & $0(0)$ \\
9 & $0(0)$ & $0(0)$ & $0(0)$ & $0(0)$ \\
10 & $0(0)$ & $0(0)$ & $0(0)$ & $0(0)$ \\
11 & $6(40.0)$ & $1(6.7)$ & $0(0)$ & $0(0)$ \\
12 & $1(6,7)$ & $1(6.7)$ & $0(0)$ & $0(0)$ \\
13 & $2(13,3)$ & $1(6.7)$ & $1(6.7)$ & $0(0)$ \\
14 & $0(0)$ & $0(0)$ & $0(0)$ & $0(0)$ \\
15 & $0(0)$ & $0(0)$ & $0(0)$ & $0(0)$ \\
16 opción a & $0(0)$ & $0(0)$ & $0(0)$ & $0(0)$ \\
16 opción b & $1(6,7)$ & $0(0)$ & $1(6.7)$ & $0(0)$ \\
16 opción c & $5(33,3)$ & $1(6.7)$ & $6(40,0)$ & $0(0)$ \\
16 opción d & $1(6,7)$ & $2(13,3)$ & $0(0)$ & $0(0)$ \\
16 opción e & $0(0)$ & $1(6.7)$ & $0(0)$ & $0(0)$ \\
17 & $0(0)$ & $0(0)$ & $0(0)$ & $0(0)$ \\
18 & $0(0)$ & $0(0)$ & $0(0)$ & $0(0)$ \\
19 & $7(46,7)$ & $1(6.7)$ & $1(6.7)$ & $0(0)$ \\
20 & $0(0)$ & $0(0)$ & $0(0)$ & $0(0)$ \\
\hline
\end{tabular}

Fuente: Elaborado por los investigadores

En los ítems 4d, 11, 16c, 19 más del 15\% de los pacientes tenían objeción o comentario. En el ítem 19 el comentario frecuente fue: "No sé qué es una bomba de insulina", al analizarlo el grupo investigador decidió dejar la pregunta cómo se redactó en la versión preliminar, para garantizar la comparabilidad de resultados con estudios realizados en otros contextos, pero se propuso indicar al evaluador que estos ítems que requieren la explicación de estos procedimientos, debe dársele al paciente si lo necesita. En la pregunta 11 ("Soy menos consciente de cuando empiezan mis hipoglucemias que antes") el 40\% de los pacientes manifestó no comprender el orden temporal de la manifestación de síntomas. El equipo investigador decidió incluir el adver- 
Tabla 3. Versión Final del instrumento HypoA-Q

\section{Cuestionario de conciencia de Hipoglucemia}

Estas preguntas son acerca de su experiencia reciente de hipoglucemia, también conocida como nivel bajo de glucosa baja en sangre. Por favor marque el cuadro de cada línea que mejor describa su experiencia. No hay respuestas correctas o incorrectas. Solo queremos averiguar sobre sus experiencias.

1. En la semana pasada, ¿qué tan a menudo ha sufrido de cualquier tipo de hipoglucemia (leve o severa, durante el día o la noche)?

veces (por favor ingrese un número)

2. En los últimos 6 meses, ¿qué tan a menudo ha sufrido de hipoglucemia en la que...

a)...haya necesitado ayuda/no fue capaz de tratarse a sí mismo?

_ veces (por favor ingrese un número)

b)...hayan sido llamados los servicios de emergencias para ayudarlo?

_ veces (por favor ingrese un número)

c)...haya sido llevado a un hospital (urgencias) para recibir tratamiento? veces (por favor ingrese un número)

d)...haya sido internado en un hospital (por una noche o más) ?

\section{A. Hipoglucemias cuando está despi- Nunca} erto

Una o dos
veces

3. En los últimos 6 meses, ¿qué tan a menudo ha tenido hipoglucemia cuando está despierto?

4. En los últimos 6 meses, ¿qué tan a menudo ha tenido hipoglucemia cuando está despierto en la que...

a)...haya tenido síntomas y fue capaz de tratarse usted mismo?

b)...haya tenido síntomas y no fue capaz 1 de tratarse usted mismo?

c)...haya necesitado que alguien más le 1 diera glucosa por vía oral?

d)...haya necesitado que alguien más le 1 administrara un medicamento inyectado?

5. En el último mes, ha tenido mediciones del nivel de glucosa en la sangre (en $\mathrm{mg} / \mathrm{dl}$ ) de...
a)...63 a 70 ?
b)...54 a 62 ?
c)...45 a 53 ?

d)...menor de 45?

6. ¿Qué tan bajo debe estar el nivel de glucosa en su sangre usualmente antes de que sienta uno de los siguientes síntomas?

a) Temblor, corazón acelerado, acaloramiento, sudoración, hambre

$\begin{array}{llllll}\text { Nunca } & \begin{array}{l}\text { Una o dos } \\ \text { veces }\end{array} & \begin{array}{l}\text { Tres o } \\ \text { cuatro } \\ \text { veces }\end{array} & \begin{array}{l}\text { Alrededor de } \\ \text { una a dos veces } \\ \text { al mes }\end{array} & \begin{array}{l}\text { Alrededor } \\ \text { de una } \\ \text { vez por } \\ \text { semana }\end{array} & \begin{array}{l}\text { Más } \\ \text { de una } \\ \text { vez por } \\ \text { semana }\end{array} \\ 1 & 2 & 3 & 4 & 5 & 6 \\ 1 & 2 & 3 & 4 & 5 & 6 \\ 1 & 2 & 3 & 4 & 5 & 6 \\ 1 & 2 & 3 & 4 & 5 & 6\end{array}$

Si la respuesta es sí, ¿qué tan a menudo presento síntomas de hipoglucemia?

$\begin{array}{llllll}\text { Sí1 No0 } & 4 & 3 & 2 & 1 & 0 \\ \text { No sabe9 } & & & & 1 & 0 \\ \text { Sí1 No0 } & 4 & 3 & 2 & 1 & 0 \\ \text { No sabe9 } & & 3 & 2 & & \\ \text { Sí1 No0 } & 4 & & & 1 & 0 \\ \text { No sabe9 } & & 3 & 2 & \end{array}$

No sabe9

$71 \mathrm{mg} / \mathrm{dL}$ o $\quad 63-70 \mathrm{mg} / \quad 54-62 \mathrm{mg} / \quad 53-45 \mathrm{mg} / \mathrm{dL} \quad$ Menor de Yo no más $\mathrm{dL} \quad \mathrm{dL} \quad 45 \mathrm{mg} / \mathrm{dL}$ tengo ninguno de estos síntomas $\mathrm{mg} / \mathrm{dL}$

9 
b) Debilidad, falta de coordinación, confusión, mareo, incapacidad de concentrarse, dificultad para hablar, visión borrosa, sueño,cansancio, irritabilidad, comportamiento raro

c) Náuseas, cosquilleo, dolor de cabeza

$$
0
$$

1

2 0

1
Nunca

7. Presentó síntomas cuando el nivel de glucosa en mi sangre es bajo.

8. Yo "simplemente sé" cuando estoy teniendo hipogluce- 4 mia por cómo me siento.

9. Reviso mi nivel de glucosa en la sangre cuando me siento mal.

10. Otras personas reconocen que estoy teniendo hipoglu- $\quad 4$ cemia antes que yo.

$\begin{array}{lllll}1 & 2 & 3 & 4 & 9 \\ \text { Nunca } & \text { Rara vez } & \text { A veces } & \begin{array}{l}\text { Frecuent- } \\ \text { emente }\end{array} & \text { Siempre } \\ 4 & 3 & 2 & 1 & 0 \\ 4 & 3 & 2 & 1 & 0 \\ 4 & 3 & 2 & 1 & 0 \\ 4 & 3 & 2 & 1 & 0 \\ \begin{array}{l}\text { Muy en } \\ \text { desacuer- } \\ \text { do }\end{array} & \begin{array}{l}\text { En desac- } \\ 0\end{array} & \begin{array}{l}\text { Ni de acuerdo o } \\ \text { endo }\end{array} & \begin{array}{l}\text { De acu- } \\ \text { erdo }\end{array} & \begin{array}{l}\text { Muy de } \\ \text { acuerdo }\end{array} \\ 0 & 1 & 2 & 3 & 4 \\ 0 & 1 & 2 & 3 & 4 \\ & 1 & 2 & 3 & 4\end{array}$

11. Ahora soy menos consciente de cuando empiezan mis hipoglucemias que antes.

12. He perdido síntomas que solía tener cuando el nivel de glucosa en mi sangre estaba bajo.

13. En los últimos 6 meses, he estado más consciente de $\quad 0$ cuando empiezan mis hipoglucemias que antes.

14. ¿Hay algo más que quisiera mencionar sobre sus hipoglucemias o su conciencia de las hipoglucemias cuando está despierto? Si ese es el caso por favor escríbalo en el cuadro

\section{A. Hipoglucemias cuando se está dormido}

15. En los últimos 6 meses, ¿qué tan a menudo ha tenido hipoglucemia cuando está dormido?

16. En los últimos 6 meses, ¿qué tan a menudo ha tenido hipoglucemia mientras está dormido en la que...

a)...haya tenido síntomas y no fue capaz 0 de tratarse usted mismo?

b)...haya tenido síntomas y haya necesi- 0 tado que alguien más le diera glucosa por vía oral?

c)... haya necesitado que alguien más le 0 administrara un medicamento inyectado?

d)... haya resultado en un problema may- 0 or (ataque, mordedura de lengua, caída, colapso, incontinencia, etc)?

e)... se quedó dormido y se dió cuenta después de que había tenido hipoglucemia?

\section{Nunca}

0

Nunca
1

\section{Menos de una vez por mes}

Alrededor de una o dos veces por mes

2

Alrededor de una o dos veces por mes una vez por mes

2

1

1

0

\section{2}

2

2
1

1

1
Alrededor de una vez por semana 3

Alrededor de una vez por semana

3

3

3

3

3
Alrededor de dos veces por semana

4

Alrededor de dos veces por semana

$$
4
$$$$
4
$$

4

4

4

4
La mayoría de días

5
5

La mayoría de días

5

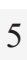

5

5 5 


\begin{tabular}{|c|c|c|c|c|c|c|}
\hline Mientras duerme & Nunca & Rara vez & A veces & Frecuentemente & Siempre & No aplica \\
\hline $\begin{array}{l}\text { 17... Presento síntomas que me despi- } \\
\text { ertan cuando el nivel de glucosa en mi } \\
\text { sangre está bajo }\end{array}$ & 4 & 3 & 2 & 1 & 0 & 9 \\
\hline $\begin{array}{l}18 \ldots \text { Otras personas reconocen que es- } \\
\text { toy teniendo hipoglucemia antes que yo }\end{array}$ & 0 & 1 & 2 & 3 & 4 & 9 \\
\hline $\begin{array}{l}\text { 19... Mi bomba de insulina/aparato de } \\
\text { monitoreo me despierta cuando el nivel } \\
\text { de glucosa de mi sangre está bajo }\end{array}$ & 0 & 1 & 2 & 3 & 4 & 9 \\
\hline
\end{tabular}

Fuente: Elaborado por los investigadores

\section{Discusión}

Dada la importancia de medir la conciencia de la hipoglucemia en pacientes diabéticos, para disminuir el riesgo de hipoglucemia severa y complicaciones (28), existen instrumentos como: el (Gold Score, el cuestionario de Clarke y el método de Pedersen-Bjergaard) que no abarcan de manera total aspectos de la hipoglucemia cuando se está despierto y dormido, lo cual significa que este es el valor agregado del instrumento HypoA-Q $(21,23,24,29)$.

El Gold Score es un sistema de puntuación que se basa en la respuesta a una sola pregunta: "¿sabe cuándo comienzan sus hipoglucemias?". Al validar esta puntuación, la hipoglucemia bioquímica se definió como menos de $54 \mathrm{mmol} /$ litro (54 mg/dL). Los resultados se expresan mediante una escala de Likert de 7 puntos, donde 1 = 'siempre consciente' y 7 = 'nunca consciente' (20); por su parte el instrumento HypoA-Q a diferencia de éste, permite la valoración de la conciencia de la hipoglucemia en el día y en la noche mientras duerme, por ello es más efectivo y tiene mayor cobertura.

Por su parte, el cuestionario de Clarke se compone de 8 preguntas que caracterizan la exposición individual a episodios de la hipoglucemia moderada y grave para evaluar el umbral glucémico y la respuesta sintomática a la hipoglucemia. La evaluación otorga un puntaje donde un valor mayor o igual a 4 indica disminución de la conciencia de la hipoglucemia $(17,22)$. A diferencia de este cuestionario, el instrumento HypoA-Q mediante 16 ítems relaciona el reporte de eventos hipoglucémicos y la utilización de medidas para cuidar la propia salud, lo cual permite identificar la presencia de episodios de la hipoglicemia y sus síntomas.

En el método de Pedersen-Bjergaard la disminución de la conciencia sobre la hipoglucemia se evalúa mediante la pregunta "¿cómo se siente cuando está bajo el nivel de glicemia?", y el encuestado selecciona una respuesta de "siempre", "generalmente", "a veces / ocasionalmente" o "nunca". La respuesta 'generalmente' implica disminución de conciencia de la hipoglucemia, mientras que las respuestas de 'a veces / ocasionalmente' o 'nunca' implican disminución severa de la misma $(23,19)$. Es decir, que el instrumento HypoA-Q tiene una ventaja adicional sobre este método, porque además de preguntar al paciente cómo se siente cuando tiene el nivel de glucosa bajo en general, es específico para cuando se está despierto y dormido.

En este sentido, HypoA-Q se constituye en un instrumento de relevancia clínica puesto que, a diferencia de los demás instrumentos, aporta información sobre la caracterización de los episodios nocturnos de la hipoglucemia y determina su gravedad, frecuencia y la utilización de servicios de salud para su tratamiento. Respecto a la hipoglucemia nocturna se evidenció que afecta potencialmente la capacidad del paciente para despertarse y responder con una ingesta adecuada de carbohidratos (30-31). En consecuencia, es importante contar con una versión en español colombiano. Este estudio es un primer paso para lograr este objetivo, al 


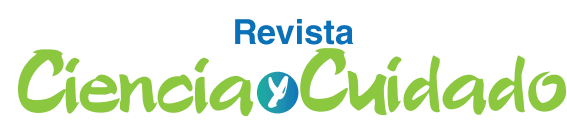

Scientific Journal of Nursing

realizar la traducción y adaptación al contexto colombiano; es por eso, que a partir de esta versión se pueden realizar los estudios sobre la evaluación de sus propiedades psicométricas. Posteriormente con el proceso de validez de contenido y de criterio del instrumento se pueden aportar datos que mejoren el instrumento.

No obstante, el instrumento en español es aún susceptible de ser mejorado cuando sea revisado y aplicado por varios profesionales de salud. Este es un aspecto a incluir como parte de la discusión.

Al mismo tiempo, la propuesta del grupo de calidad de vida de la EORTC para realizar este tipo de estudios permitió obtener una versión en español de la escala que cumpliera para cada ítem los criterios de: comprensión, ausencia de palabras difíciles u ofensivas. Se observó que dos ítems usan lenguaje técnico que podría afectar la comprensión en algunos pacientes, concretamente: el ítem 16.c: "en los últimos seis meses, ¿qué tan a menudo ha tenido hipoglucemia mientras está dormido en la que haya necesitado que alguien más le diera una inyección de glucagón” y el ítem 19: "mi bomba de insulina/aparato de monitoreo me despierta cuando el nivel de glucosa de mi sangre está bajo". Este problema se presenta dado que las preguntas se enfocan en aspectos clave de la atención, razón por la cual es necesario explicarlas al encuestado antes de aplicar la escala.

De donde se infiere que para garantizar la calidad de la medición de la conciencia sobre la hipoglucemia, en la aplicación del instrumento es necesario formular los ítems, utilizando el lenguaje con el cual se encuentra descrito cada uno; por ello, se sugiere que la aplicación del mismo la realice un evaluador que esté capacitado para explicar las palabras técnicas al paciente.
Validación transcultural del HypoA-Q para medir conciencia de hipoglucemia en pacientes diabéticos

\section{Conclusiones}

A partir de esta traducción de la escala se propone realizar la evaluación de sus propiedades psicométricas, empleando tanto la teoría clásica de los test como la teoría de la respuesta al ítem. De ahí que sea necesario emplear: el primer modelo de medición para determinar la confiabilidad y validez de la escala; y, el segundo para determinar el comportamiento de cada una de las preguntas, así como de las opciones de respuesta. En definitiva, este estudio permite en la práctica clínica identificar la disminución de la conciencia de la hipoglucemia en el paciente cuando está despierto y dormido; por tanto, puede reducir los casos de la hipoglucemia severa y tener en cuenta su uso en guías de manejo para pacientes diabéticos en Colombia. Además, el contar con un instrumento validado facilita el desarrollo de estudios relacionados con la hipoglicemia.

\section{Agradecimientos}

Los autores agradecen a: la Doctora Jane Speight, autora del instrumento, quien autorizó el uso del mismo para el estudio de adaptación transcultural y validación; así como a la docente María Teresa Aparicio, especialista en enseñanza de la lengua inglesa de la Universidad de Cambridge quien contribuyó en la revisión de las traducciones inversas y su consolidación; al igual que a los pacientes del Hospital San José, por su participación en la prueba piloto del instrumento.

\section{Conflicto de Intereses}

Los autores declaran no tener ningun conflicto de intereses.

\section{Referencias Bibliográficas}

1. Cuenta de Alto Costo. Situación de la enfermedad renal crónica, la hipertensión arterial y la diabetes mellitus en Colombia 2019 [Internet]. CAC. 2018 [cited 2021 Jun 5]. p. 65-79. Available from: https://cuentadealtocosto.org/site/wpcontent/uploads/2020/06/CAC.Co_2020 06_24_Libro_Sit_ERC 2019V7.pdf

2. Kuroda N, Kusunoki Y, Osugi K, Ohigashi M, Azuma D, Ikeda H, et al. Relationships between time in range, glycemic variability including hypoglycemia and types of diabetes therapy in Japanese patients with type 2 diabetes mellitus: Hyogo Diabetes Hypoglycemia Cognition Complications study. J Diabetes Investig. 2021 Feb;12(2):244-253. doi: https://doi.org/10.1111/jdi.13336

3. Bakar A, Qomariah SN, Santoso CH, Gustomi MP, Syaful Y, Fatmawa L. Factors the incidence of hypoglycemia in diabetes mellitus patients: A pilot study in the emergency room. Enferm Clin. 2020 Jun;30 Suppl 
5:46-49. English, Spanish. doi: https://doi.org/10.1016/j.enfcli.2019.11.020

4. Yue X, Wu J, Ruan Z, Wolden ML, Li L, Lin Y. The Burden of Hypoglycemia in Patients With Insulin-Treated Diabetes Mellitus in China: Analysis of Electronic Medical Records From 4 Tertiary Hospitals. Value Health Reg Issues. 2020 May;21:17-21. doi: https://doi.org/10.1016/j.vhri.2019.06.003

5. Heller SR, Peyrot M, Oates SK, Taylor AD. Hypoglycemia in patient with type 2 diabetes treated with insulin: it can happen. BMJ Open Diabetes Res Care. 2020 Jun;8(1):e001194. doi: https://doi.org/10.1136/ bmjdrc-2020-001194

6. Freeland B. Hypoglycemia in Diabetes Mellitus. Home Healthc Now. 2017 Sep;35(8):414-419. doi: https:// doi.org/10.1097/NHH.0000000000000584

7. Ratner RE. Hypoglycemia: New Definitions and Regulatory Implications. Diabetes Technol Ther. 2018;20(S2).

8. Summary of Revisions: Standards of Medical Care in Diabetes-2018. Diabetes Care. 2018;41(s1):s1-2.

9. Emral R, Pathan F, Cortés CAY, El-Hefnawy MH, Goh SY, Gómez AM, Murphy A, Abusnana S, Rudijanto A, Jain A, Ma Z, Mirasol R; IO HAT Investigator Group. Self-reported hypoglycemia in insulin-treated patients with diabetes: Results from an international survey on 7289 patients from nine countries. Diabetes Res Clin Pract. 2017 Dec;134:17-28. doi: https://doi.org/10.1016/j.diabres.2017.07.031

10. Pieber TR, Marso SP, McGuire DK, Zinman B, Poulter NR, Emerson SS, et al. DEVOTE 3: temporal relationships between severe hypoglycaemia, cardiovascular outcomes and mortality. Diabetologia. 2018 Jan;61(1):58-65. doi: https://doi.org/10.1007/s00125-017-4422-0

11. Cha SA, Yun JS, Lim TS, Hwang S, Yim EJ, Song KH, Yoo KD, Park YM, Ahn YB, Ko SH. Severe Hypoglycemia and Cardiovascular or All-Cause Mortality in Patients with Type 2 Diabetes. Diabetes Metab J. 2016 Jun;40(3):202-10. doi: https://doi.org/10.4093/dmj.2016.40.3.202

12. International Hypoglycaemia Study Group. Hypoglycaemia, cardiovascular disease, and mortality in diabetes: epidemiology, pathogenesis, and management. Lancet Diabetes Endocrinol. 2019 May;7(5):385-396. doi: https://doi.org/10.1016/S2213-8587(18)30315-2

13. 13. Umpierrez G, Korytkowski M. Diabetic emergencies - ketoacidosis, hyperglycaemic hyperosmolar state and hypoglycaemia. Nat Rev Endocrinol. 2016Apr;12(4):222-32. doi: https://doi.org/10.1038/nrendo.2016.15

14. Jensen MH, Dethlefsen C, Hejlesen O, Vestergaard P. Association of severe hypoglycemia with mortality for people with diabetes mellitus during a 20-year follow-up in Denmark: a cohort study. Acta Diabetol. 2020 May;57(5):549-558. doi: https://doi.org/10.1007/s00592-019-01447-x

15. Freeman J. Management of hypoglycemia in older adults with type 2 diabetes. Postgrad Med. 2019 May;131(4):241-250. doi: https://doi.org/10.1080/00325481.2019.1578590

16. Kana Kadayakkara D, Balasubramanian P, Araque K, Davis K, Javed F, Niaki P, Majumdar S, Buller G. Multidisciplinary strategies to treat severe hypoglycemia in hospitalized patients with diabetes mellitus reduce inpatient mortality rate: Experience from an academic community hospital. PLoS One. 2019 Aug 8;14(8):e0220956. doi: https://doi.org/10.1371/journal.pone.0220956

17. Frier BM. Impaired Awareness of Hypoglycaemia. In: Hypoglycaemia in Clinical Diabetes [Internet]. 2007. p. 141-70. (Wiley Online Books). Available from: https://doi.org/10.1002/9780470516270.ch7

18. Henderson JN, Allen KV, Deary IJ, Frier BM. Hypoglycaemia in insulin-treated Type 2 diabetes: frequency, symptoms and impaired awareness. Diabet Med. 2003 Dec;20(12):1016-21. doi: https://doi.org/10.1046/ j.1464-5491.2003.01072.x

19. Martín-Timón I, Del Cañizo-Gómez FJ. Mechanisms of hypoglycemia unawareness and implications in diabetic patients. World J Diabetes. 2015 Jul 10;6(7):912-26. doi: https://doi.org/10.4239/wjd.v6.i7.912

20. Gold AE, MacLeod KM, Frier BM. Frequency of severe hypoglycemia in patients with type I diabetes with 
impaired awareness of hypoglycemia. Diabetes Care. 1994 Jul;17(7):697-703. doi: https://doi.org/10.2337/ diacare.17.7.697

21. Jansa M, Quirós C, Giménez M, Vidal M, Galindo M, Conget I. Análisis psicométrico de las versiones en lengua castellana y catalana de un cuestionario de percepción de la hipoglucemia. Med Clin (Barc). 2015;10:440-4.

22. Clarke WL, Cox DJ, Gonder-Frederick LA, Julian D, Schlundt D, Polonsky W. Reduced awareness of hypoglycemia in adults with IDDM. A prospective study of hypoglycemic frequency and associated symptoms. Diabetes Care. 1995 Apr;18(4):517-22. doi: https://doi.org/10.2337/diacare.18.4.517

23. Pedersen-Bjergaard U, Agerholm-Larsen B, Pramming S, Hougaard P, Thorsteinsson B. Activity of angiotensin-converting enzyme and risk of severe hypoglycaemia in type 1 diabetes mellitus. Lancet. 2001;357(9264):1248-53.

24. Speight J, Barendse SM, Singh H, Little SA, Inkster B, Frier BM, et al. Characterizing problematic hypoglycaemia: Iterative design and preliminary psychometric validation of the Hypoglycaemia Awareness Questionnaire (HypoA-Q). Diabet Med. 2016;33(3):376-85.

25. Kuliś D, Bottomley A, Velikova G, Greimel E, Koller M, EORTC, et al. EORTC Quality of Life Group Translation Procedure [Internet]. Brussels: EORTC. 2017. p. 24. Available from: https://www.eortc.org/app/ uploads/sites/2/2018/02/translation_manual_2017.pdf

26. Real Academia Española. Diccionario de la lengua española, 23 ed., [versión 23.3 en línea] [Internet]. 2014 [cited 2019 Jun 15]. Available from: https://dle.rae.es

27. 27. American Diabetes Association. 6. Glycemic targets: Standards of Medical Care in Diabetes 2021. Diabetes Care 2021; 44(Suppl. 1):S73-S84

28. Carrillo Algarra AJ, Beltrán KM, Bolivar Castro DM, Hernández Zambrano SM, Henao Carrillo DC. Cuidados de enfermería para la persona adulta, diabética con hipoglucemia: revisión integrativa . Repert. Med. Cir. $2021 ; 59-3$.

29. Flatt AJS, Little SA, Speight J, Leelarathna L, Walkinshaw E, Tan HK et al. Predictors of Recurrent Severe Hypoglycemia in Adults With Type 1 Diabetes and Impaired Awareness of Hypoglycemia During the HypoCOMPaSS Study. Diabetes Care. 2020 Jan;43(1):44-52. doi: 10.2337/dc19-0630. Epub 2019 Sep 4. PMID: 31484666.

30. Ryan EA, Shandro T, Green K, Paty BW, Senior PA, Bigam D, et al. Assessment of the Severity of Hypoglycemia and Glycemic Lability in Type 1 Diabetic Subjects Undergoing Islet in Transplantation. Diabetes. 2004;53(4):955-62.

31. Duncan E, Fitzpatrick D,Ikegwuonu T, Evans J, Maxwell M. Role and prevalence of impaired awareness of hypoglycaemia in ambulance service attendances to people who have had a severe hypoglycaemic emergency: a mixed-methods study. BMJ Open. 2018; 8(4): e019522. doi: https://doi.org/10.1136/ bmjopen-2017-019522 